\title{
Radiografía periapical como herramienta en el diagnóstico y tratamiento de quiste periapical
}

\section{Periapical radiography such as a tool in the diagnosis and treatment of periapical cyst}

Verbel Bohórquez J*, Ramos Manotas J**, Díaz Caballero A***

\section{RESUMEN}

Introducción: El quiste periapical es una lesión inflamatoria común de los maxilares, que al alcanzar un tamaño significativo, causa deformidad del hueso afectado, retención o incluso el desplazamiento de dientes adyacentes. Se presentó caso de paciente que acudió a consulta odontológica, por presencia de sintomatología dolorosa durante el consumo de alimentos. Al examen clínico se observó encía adherida con presencia de inflamación y enrojecimiento a nivel de órgano dentario 14; radiográficamente se evidenció imagen radiolúcida unilocular, bien definida, abarcando dicho órgano dentario. La lesión fue eliminada quirúrgicamente con la posterior aplicación de material sellador. En el control postoperatorio se observó adecuado proceso de cicatrización y ausencia de sintomatología.

Discusión: Se reporta caso de quiste periapical, que ocupaba el periápice de órgano dentario posterosuperior derecho. Radiográficamente se evidenció variabilidad mínima en el tamaño de la imagen. Se describe su resección quirúrgica y se informan los hallazgos de histopatología. En la revisión bibliográfica realizada se determinó la importancia de la elección de las radiografías periapicales en el área de endodoncia, y su papel en el diagnóstico, planificación de tratamiento y seguimiento de las piezas dentarias y tejidos circundantes comprometidos, pese a los inconvenientes de la técnica radiográfica.

Palabras clave: Quiste radicular, rayos X, procedimientos quirúrgicos operativos.

\section{SUMMARY}

Introduction: The periapical cyst is a common inflammatory lesion of the jaws. When the cyst reaches a significant size, it cause a deformity of the affected bone, retention or displacement of adjacent teeth. The case of a patient was presented who went to dental appointment because the presence of painful symptomatology during food consumption. A clinical examination revealed that gingiva was red and swollen at tooth 14; radiographically evidenced a unilocular radiolucency which covered the dental organ. The lesion was excised surgically with the subsequent application of sealant material. After surgery, was evidenced healing and the patient was no longer in pain.

Discussion: We report a case of periapical cyst, which occupied the dental organ periapex upper right posterior. Radiographically evidenced minimal variability in the size of the image. Its resection is described and we inform the histopathological findings. In the literature review we found the importance of the choice of periapical $\mathrm{x}$-rays

* Odontóloga. Universidad de Cartagena. Joven Investigador Colciencias.

** Odontólogo. Universidad de Cartagena. Magíster en Endodoncia, Universidad de San Luis Potosí (México). Profesor titular, Universidad de Cartagena.

*** Odontólogo. Universidad de Cartagena. Especialista en Periodoncia Universidad Javeriana. Magíster en educación Universidad del Norte. Candidato a Doctor en Ciencias Biomédicas Universidad de Cartagena. Profesor titular Universidad de Cartagena. Director Grupo de Investigaciones GITOUC. 
in the endodontic area, and its role in the diagnosis, treatment planning and monitoring of the teeth and surrounding tissues involved, despite the drawbacks of traditional radiographic technique.

Key words: Radicular cyst, X-rays, surgical procedures operative.

Fecha de recepción: 25 de marzo de 2014.

Aceptado para publicación: 10 de abril de 2014.

Verbel Bohórquez J, Ramos Manotas J, Díaz Caballero A. Radiografía periapical como herramienta en el diagnóstico y tratamiento de quiste periapical. Av. Odontoestomatol 2015; 31 (1): 25-29.

\section{INTRODUCCIÓN}

El quiste periapical es reconocido como la lesión quística de origen inflamatorio, de mayor frecuencia entre los quistes de origen odontogénicos, la que oscila entre el 70 al $75 \%$ de los casos (1). Este se desarrolla en el periápice de una pieza dentaria no vital donde existe proliferación de los restos epiteliales de malassez estimulada por la inflamación e infección bacteriana desarrollada en la pulpa dentaria (2). Está demostrado que ocurren a cualquier edad, sobre todo en la tercera década, sin predilección por el sexo (3).

Los quistes radiculares son cavidades patológicas de contenido líquido, semilíquido, sólido o gaseoso, revestidas de epitelio en su cara interior y por tejido conectivo en el exterior (4). En general, todos los quistes odontogénicos suelen ser asintomáticos en sus etapas iniciales, a menos que se infecten secundariamente o alcancen un tamaño significativo (5).

Es importante llevar a cabo un examen clínico y radiográfico del diente para la elección del tratamiento de la lesión radicular. Hay dos opciones de tratamiento: el retratamiento endodóntico no quirúrgico de la pieza dentaria, y la cirugía apical. Se recurre al segundo en casos de: signos y síntomas como dolor, inflamación, fístula o de complicaciones anatómicas como conductos calcificados, cálculos pulpares, curvas radiculares no superables; errores en el procedimiento como perforaciones, sobreinstrumentación, sobreextensión e incluso fractura de instrumentos, así como la persistencia de enfermedad apical luego de un tratamiento endodóntico convencional (6).
Para la planificación precisa de cirugía endodóntica y, un resultado predecible del procedimiento, es crucial determinar las dimensiones exactas y ubicación de la lesión periapical, así como su relación con las raíces y otras estructuras anatómicas vecinas (7). Esto se realiza por medio de radiografías periapicales, las cuales a pesar de presentar limitaciones propias de la técnica representando bidimensionalmente estructuras tridimensionales, y al verse afectada la interpretación de las lesiones debido a las variaciones anatómicas de la región periapical, densidad de hueso, la angulación de rayos $\mathrm{X}$, de contraste radiográfico, y la ubicación real de la lesión periapical; son imprescindibles para definir hipótesis de diagnóstico y establecer el plan de tratamiento. De tal forma se requiere un examen minucioso por parte del profesional para obtener éxito completo en el tratamiento indicado (8).

\section{PRESENTACIÓN DE CASO CLÍNICO}

Paciente masculino de 55 años, que acude a consulta por presentar dolor y malestar de intensidad moderada durante el consumo de alimentos, desde hace aproximadamente 3 meses. El paciente no reportó ningún antecedente sistémico de importancia. Al examen intraoral se observó a nivel de órgano dentario (OD) 14 corona individual metal-cerámica y enrojecimiento e inflamación de encía adherida. A la palpación y percusión manifestó sensibilidad. Al examen radiográfico con radiografía periapical de órgano dentario (OD) 14 a 17 se observó radiopacidad correspondiente a material de obturación en el OD 14 y núcleo colado en ambas raíces radiculares. A nivel de dicho OD se evidenció espacio del ligamento periodontal ensanchado y, presencia de una ima- 
gen radiolúcida unilocular en forma de pera de tamaño aproximado de $8 \mathrm{~mm}$, bien definida (Fig. 1). Teniendo en cuenta los hallazgos clínicos y radiográficos descritos, el diagnóstico diferencial inicial de esta lesión incluyó quiste residual, u otro quiste en desarrollo o tumor odontogénico.

Se informó al paciente sobre las complicaciones y efectos adversos del procedimiento, así como del anonimato y confidencialidad de sus datos personales. Previa autorización y firma del consentimiento informado, se efectuó cirugía bajo anestesia local con lidocaína $2 \%$, se diseñó colgajo triangular con conservación de las papilas, disección de colgajo de espesor total, seguido de osteotomía con fresa redonda diamantada número 4 . Se llevó a cabo desbridamiento de tejido patológico, el cual, presentó un tamaño anatómico mayor al estimado con la radiografía inicial, posteriormente fue llevado a estudio histopatológico. Se realizó apicectomía de aproximadamente $3 \mathrm{~mm}$. Posterior a esto, se procedió a raspado y pulido del proceso periapical y utilización de gasa y aspirador para secar la cavidad. Se realizó obturación retrógrada con mineral trióxido agregado (MTA) y limpieza e irrigación con suero fisiológico, luego, los bordes del colgajo se reposicionaron con puntos simples aislados con Nylon 5-0. Una vez finalizada la intervención quirúrgica se le prescribió al paciente: Amoxicilina, cápsulas 500 mg por 7 días;

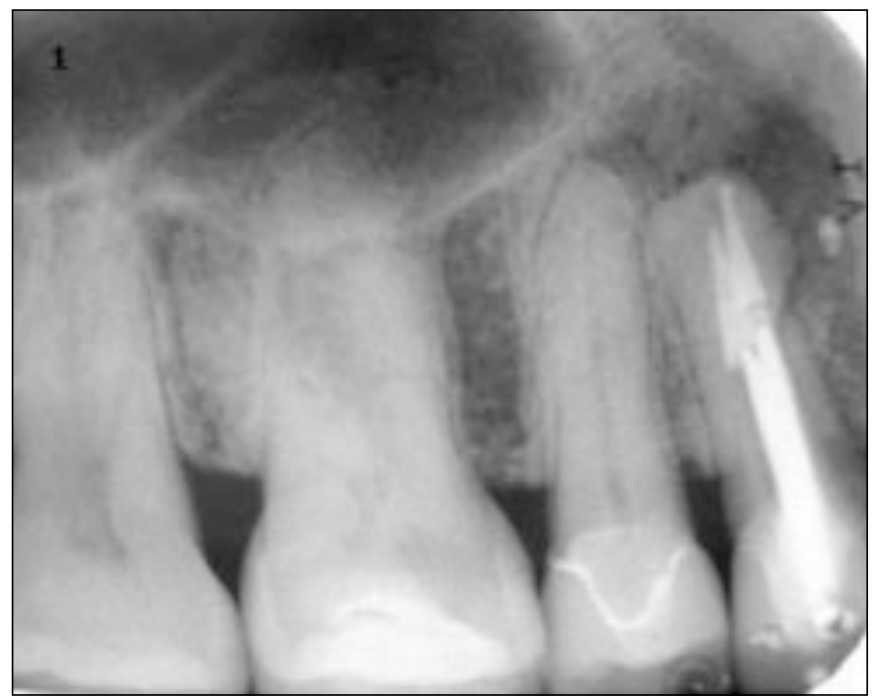

Fig. 1. Radiografía periapical preoperatoria en la que se aprecia lesión radiolúcida en el periápice de órgano dentario 14 .
Nimesulide, tabletas $100 \mathrm{mg}$ por 5 días y enjuagues con clorhexidina al $0,12 \% 2$ veces al día por 7 días. Por último, se realizó control radiográfico inmediato, en el cual se evidenció la cantidad real de pérdida ósea, que no fue identificada previamente al acto quirúrgico (Fig. 2). Las suturas se retiraron al cabo de 7 días.

El estudio histopatológico diagnosticó pared de quiste radicular; dicho estudio, mostró epitelio escamoso estratificado no queratinizado con tejido conectivo subyacente fibroso, denso, bien vascularizado y con infiltrado inflamatorio (Fig. 3). Tres meses posteriores a la remoción de la pared de la lesión, así como al sellado apical apropiado, la paciente acudió a control postoperatorio y negó sintomatología dolorosa, así como sensibilidad a la palpación o a la percusión vertical de la pieza dentaria involucrada; se observó lesión periapical en proceso de reparación, con formación de tejido óseo, lo que permitió establecer un pronóstico favorable para dicho órgano dentario (Fig. 4).

\section{DISCUSIÓN}

En el presente caso, el tamaño de la lesión, la presencia de sintomatología dolorosa, así como la persistencia de la lesión apical luego del tratamiento

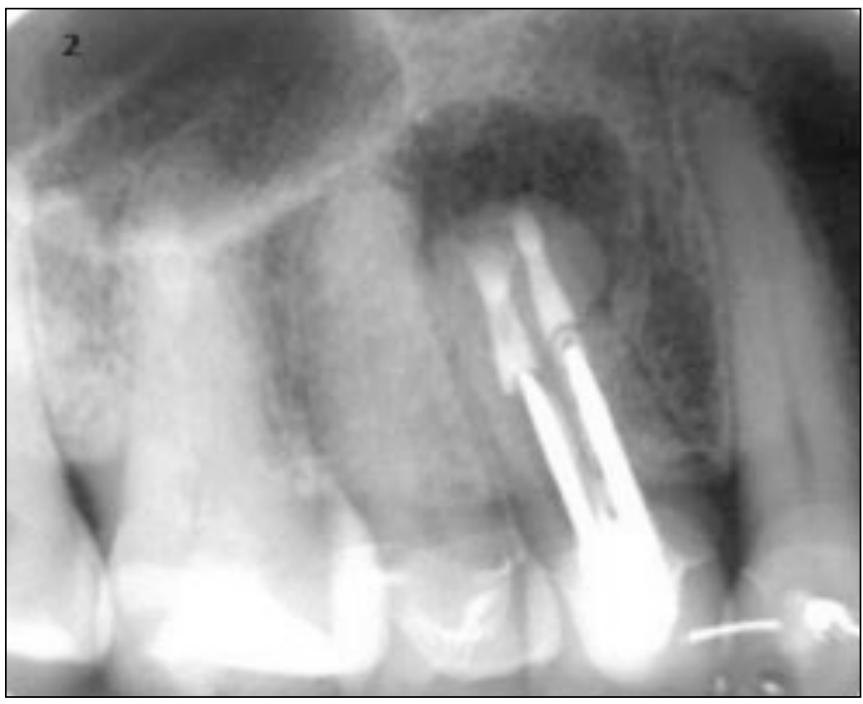

Fig. 2. Radiografía de control postoperatorio. Se evidencia pérdida ósea real y obturación con material mineral trióxido agregado. 


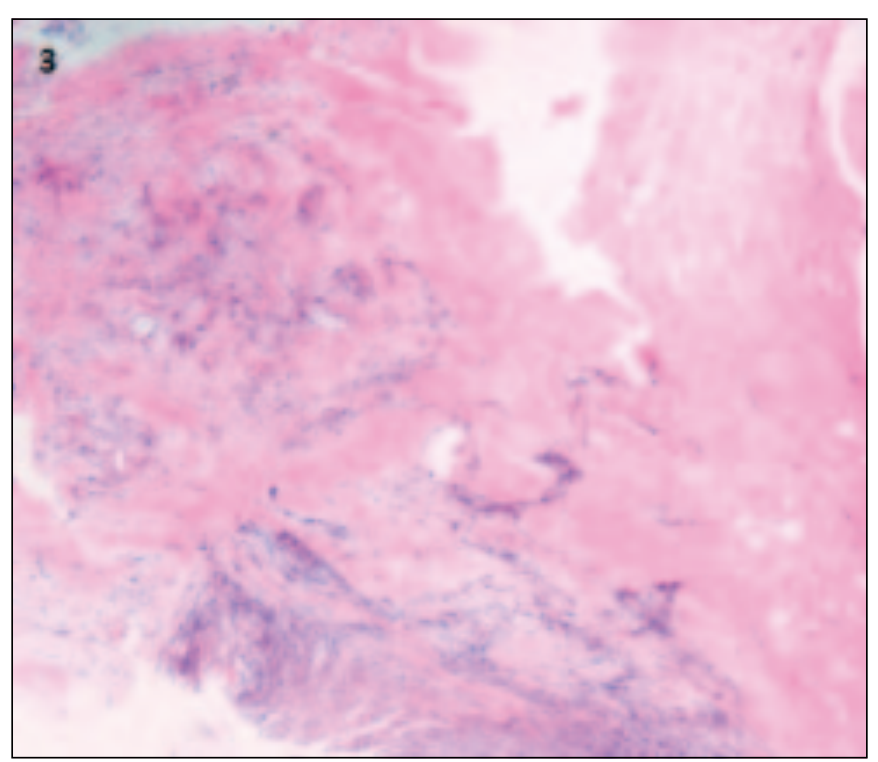

Fig. 3. Vista histológica de quiste radicular.

endodóntico, fundamentaron la realización de la cirugía apical. Tal como lo reportan Peñarrocha et al en su estudio quienes señalan que la cirugía periapical está indicada en casos de lesiones radiotransparentes mayores a $3 \mathrm{~mm}$ de diámetro, y en el resto de casos en presencia de sobreobturación y dolor (9). Thomas von Arx afirma que el objetivo de la cirugía apical es mantener una pieza dentaria por medio

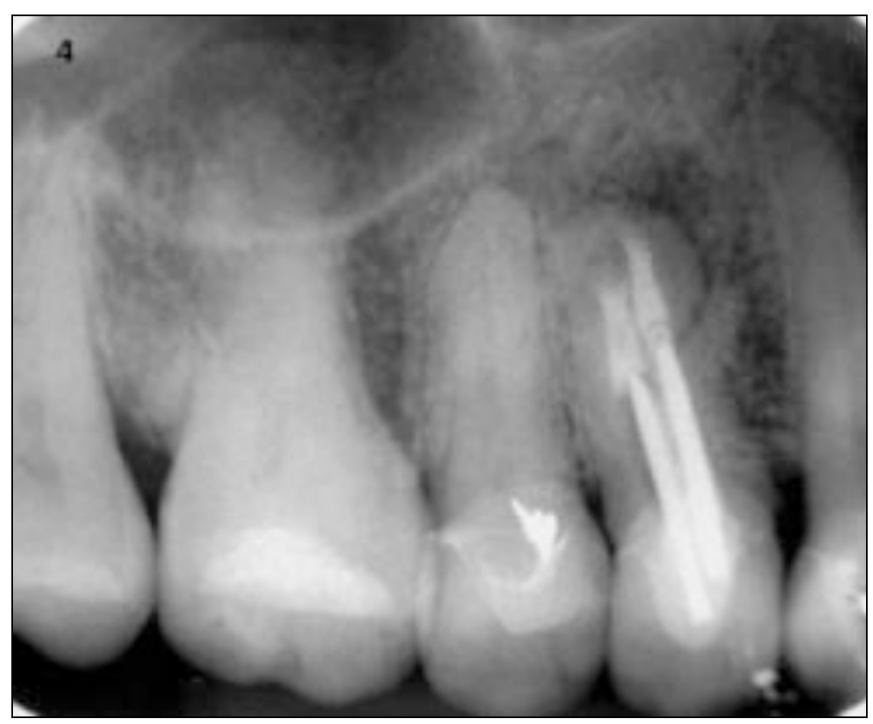

Fig. 4. Radiografía de control a los 3 meses después de cirugía endodóntica. Se aprecia notable formación de tejido óseo y disminución en el tamaño del área radiolúcida. quirúrgico debido a que la lesión no pudo ser resuelta por tratamiento endodóntico convencional (10).

Kouhyar y Mahesh obtuvieron como resultado que la región canina-premolar de ambas arcadas dentarias expresan la mayor cantidad de distorsión angular y variabilidad en las radiografías (11). Similar a los hallazgos de Patel et al quienes reportan que debido a la complejidad del esqueleto maxilofacial, las imágenes radiográficas no siempre reproducen con precisión la anatomía que está siendo evaluada (12). Lo anterior se corrobra en el presente caso clínico, ya que luego del procedimiento quirúrgico y del desbridamiento de la patología, se observó que el tamaño anatómico de la lesión fue indudablemente mayor a lo determinado inicialmente por el tamaño de la imagen radiográfica ubicada en la zona premolar derecha.

Las imágenes obtenidas mediante radiografías convencionales intraorales revelan una información en dos dimensiones (altura y anchura), lo que limita en el diagnóstico y por tanto en el plan de tratamiento (13). En contraste, Cedeño et al afirman que las radiografías periapicales a pesar de su margen de error en cuanto a la longitud en milímetros de $6,75 \%$ para la arcada superior sin distinción de lados (derecho e izquierdo) y de $4,4 \%$ para la arcada inferior sin distinción de lados (derecho e izquierdo), siguen siendo el método más certero para el diagnóstico y control de la longitud, angulaciones y paralelismo radicular, en comparación con otras radiografías como las panorámicas (14). Concordante con lo reportado en el presente caso clínico, en donde, a pesar de la discrepancia entre el tamaño radiográfico y anatómico, los síntomas del paciente y la adecuada experiencia del profesional clínico en la interpretación radiográfica, no limitó la selección del procedimiento ideal.

En conclusión, las radiografías periapicales son imprescindibles en los procedimientos endodónticos, pues proporcionan una información detallada de la zona perirradicular, necesaria para determinar los límites de la lesión dando una visión específica al profesional para su conveniente abordaje quirúrgico. Sin embargo, existen nuevas técnicas imagenológicas que aportan mayor especificidad pues reproducen en tres dimensiones el sitio de estudio, como lo es la tomografía axial computarizada. 


\section{AGRADECIMIENTOS}

Los autores agradecen al departamento administrativo de ciencia, tecnología e innovación COLCIENCIAS.

\section{BIBLIOGRAFÍA}

1. Balasundaram A, Shah P, Hoen mm, Wheater MA, Bringas JS, Gartner A, Geist JR. Comparison of Cone-Beam Computed Tomography and Periapical Radiography in Predicting Treatment Decision for Periapical Lesions: A Clinical Study. International journal of dentistry 2012;2012(8): $1-8$.

2. Concha G. Radiología de quistes de los maxilares. Revista Hospital Clínico Universidad de Chile 2002;13(1):23-32.

3. Varma N, Nallanchakrava S, Muppa R, Dandempally A, Panthula P. Conservative Approach in the Management of Radicular Cyst in a Child: Case Report. Case Reports in Dentistry 2013;2013(1): 1-3.

4. Vázquez D, Balsamo MF, Gandini P, Valdez A, Carbajal E. Quiste periapical inflamatorio. Revista Asociación Dental Mexicana 2009;65(1):24-7.

5. Mosqueda A, Irigoyen ME, Diaz MA, Torres MA. Quistes odontogénicos. Análisis de 856 casos. Medicina Oral 2002;7(2):89-96.

6. Osorio GI, Barcha DA, Diaz AJ, Covo E. Retratamiento endodóntico como primera elección ante cirugía apical. DUAZARY 2009;6(2):147-53.

7. Tanomaru-Filho M, Lima RK, Nakazone PA, Tanomaru JM. Use of computerized tomography for diagnosis and follow-up after endodontic surgery: clinical case report with 8 years of followup. Oral Surgery, Oral Medicine, Oral Pathology, Oral Radiology, and Endodontology 2010;109 (4):629-33.
8. Dos Anjos ML, Pereira K, Dos Anjos A, Salazar JR, Cunha FL. Evaluacion de la calidad de las radiografias periapicales obtenidas en la clinica de endodoncia por alumnos de pré-grado. Acta Odontológica Venezolana 2011;49(4):1-12.

9. Peñarrocha M, Martí E, García B, Gay C. Relationship of periapical lesion radiologic size, apical resection, and retrograde filling with the prognosis of periapical surgery. Journal of oral and maxillofacial surgery 2007;65(8):1526-9.

10. Von Arx T. Apical surgery: A review of current techniques and outcome. The Saudi Dental Journal 2011;23(1):9-15.

11. Koushyar KJ, Mahesh L. Comparación entre radiografías tradicionales y tridimensionales en odontología. Odontologia actual 2011;8(103):614.

12. Patel S, Dawood A, Whaites E, Pitt Ford T. New dimensions in endodontic imaging: part 1 . Conventional and alternative radiographic systems. International endodontic journal 2009;42(6):44762.

13. Villaverde A, Garrido P, Vera C, Rodriguez N. Cone beam computed tomography en endodoncia $(\mathrm{y}$ II). Dental practice report 2012;7(4):37-40.

14. Cedeño GA, Casasa A, Gurrola B. Comparación de las técnicas radiográficas periapical y panorámica en premolares. Revista mexicana de odontología clinica 2007;1(10):10-6.

\section{CORRESPONDENCIA}

Antonio Díaz Caballero

Facultad de Odontología

Universidad de Cartagena

Campus de la Salud Zaragocilla

Cartagena, Bolívar, Colombia

Correo electrónico: adiazc1@unicartagena.edu.co 